\title{
Malaria Vaccine: The Role of Anopheline Saliva
}

Hamad $\mathrm{MNM}^{1^{*}}$

${ }^{1}$ Head of Medical Parasitology Department, Faculty of Medical Laboratory Sciences, Elsheikh Abdallah Elbadri University

Corresponding Author: Mosab Nouraldein Mohammed Hamad

Address: Head of Medical Parasitology Department, Faculty of Medical Laboratory Sciences, Elsheikh Abdallah Elbadri University, Sudan; E-mail: musab.noor13@gmail.com

Received date: 12 February 2020; Accepted date: 06 March 2020; Published date: 15 March 2020

Citation: Hamad MNM. Malaria Vaccine: The Role of Anopheline Saliva. J Health Care and Research. 2020 Mar o6;1(1):12-13.

Copyright (c) 2020 Hamad MNM. This is an open-access article distributed under the Creative Commons Attribution License, which permits unrestricted use, distribution, and reproduction in any medium, provided the original work is properly cited.

\section{Abstract}

Malaria is one of the most spread parasitic diseases in the world, certainly, in tropical areas, it kills millions of people in those parts of the world chiefly pregnant ladies and young children.

several efforts attempt to control malaria in these areas, but it faced by many factors such as wars, vector resistance to insecticides, poor knowledge and practice of health care workers and the absence of good communications between international organizations and policymakers in that regions.

Many attempts to produce an effective vaccine against malaria failed due to looking to the parasitic cycle from one side and regardless of the sexual cycle within Anopheles mosquito. I suggest that the key to the malaria vaccine starts from the salivary gland of Anopheles mosquito.

This paper is my word to heath organizations and medical research centers to support my view to gain a future vaccine to the malaria parasite.

\section{Keywords}

Malaria Parasite; Vaccine; Saliva

\section{Background}

Malaria is a widespread parasitic disease it affects many people around the globe, particularly in tropic and subtropical regions, due to the suitability of tropical environment to vector living, breeding, and multiplication.

Most affected people in malaria infested districts are children and pregnant women, due to their low level of immunity and due to iron intake in the case of pregnant women, which is suggested to increase the risk of malaria, especially vivax malaria.

Prevalence of malaria infection in poor regions of the world supports the dissemination of disease into vast areas, due to inadequate control measures, absence of well-established research centers, civil wars, personal behavior and low income of malaria infested zones.

In addition to poor knowledge and inadequate medical professionals in that region of the world, 
Citation: Hamad MNM. Malaria Vaccine: The Role of Anopheline Saliva. J Health Care and Research. 2020 Mar 06;1(1):12-13.

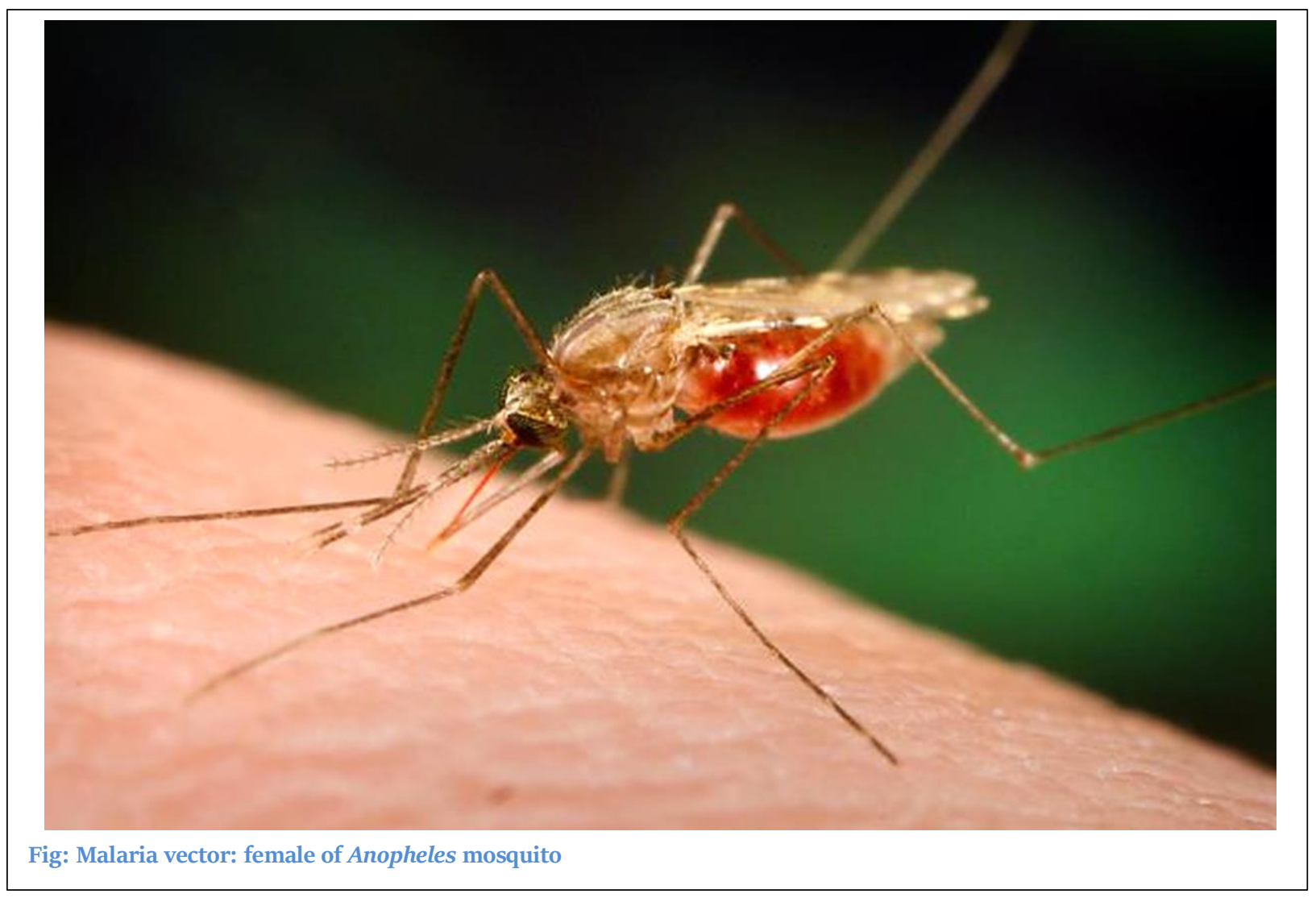

some of them have no even communication skills with the patients. Then collaboration between policymakers, world health organizations, and local health care professionals is the first step to fight malaria and to resist its dissemination to malaria-free areas.

Malaria vaccine is very essential factor to stop malaria infection, although of many attempts occur by many international medical companies and certain charity medical groups, but all of these efforts failed, due to genetic complexity of malaria parasite and due to focusing of that effort toward the erythrocytic cycle in human and neglecting the biology of the parasite in the definitive host (female of Anopheles mosquito).

I suggest that when we study the nature (anatomy, physiology, biochemistry) of the salivary gland of the definitive host, we will succeed to produce a vaccine to this fatal parasitic disease.This point of view needs a well-established laboratory and skillful group of anatomists, physiologists, biochemists, parasitologist and immunologists to start this scheme of malaria vaccination.

This paper is just a call for international organizations and medical research centers to begin the war against plasmodium species.

Gathering of views, collecting of efforts will save millions of people from malaria and its severe complications which is range from anemia, hypoglycemia to coma and death. 